\title{
Penaeus monodon GILT enzyme restricts WSSV infectivity by reducing disulfide bonds in WSSV proteins
}

\author{
Jaturon Thipwong ${ }^{1}$, Hirun Saelim ${ }^{1}$, Tanate Panrat ${ }^{2}$, Amornrat Phongdara ${ }^{1, *}$ \\ ${ }^{1}$ Department of Molecular Biotechnology and Bioinformatics, Faculty of Science, Prince of Songkla University, Hat Yai, \\ Songkhla, 90112, Thailand \\ ${ }^{2}$ Digital Media Program, Prince of Songkla University International College, Prince of Songkla University, Hat Yai, \\ Songkhla, 90112, Thailand
}

\begin{abstract}
Gamma-interferon-inducible lysosomal thiol reductase (GILT) is involved in the adaptive immune response via its effects on major histocompatibility complex (MHC)-restricted antigen presentation. In addition to antigen presentation, GILT exerts its antiviral activity by reducing disulfide bonds in proteins involved in viral infection and assembly, thereby inhibiting viral envelope-mediated infection and viral progeny production. In black tiger shrimp, Penaeus monodon GILT (PmGILT) was cloned and characterized, and found to be involved in the shrimp innate immune response and to exert neutralizing activity against white spot syndrome virus (WSSV) infection. However, the anti-WSSV mechanism of PmGILT in the shrimp innate immune response has not been defined. To explore the anti-WSSV activity of PmGILT, a yeast 2-hybrid (Y2H) assay was performed to identify WSSV proteins targeted by PmGILT. The assay revealed 4 potential PmGILT-interacting WSSV proteins: WSSV002, WSSV164, WSSV189, and WSSV471. Three of these 4 WSSV proteins (WSSV002, WSSV164 and WSSV189) were successfully produced and confirmed to interact with PmGILT in in vitro pull-down assays. WSSV189 and WSSV471 were previously identified as structural proteins, whereas WSSV164 is an immediate-early protein which has anti-melanization activity, and WSSV002 is an unknown. Because of the thiol reductase activity of PmGILT, WSSV164 and WSSV189, both of which are cysteine-containing WSSV proteins, were chosen for disulfide bond reduction assays. PmGILT reduced intrachain disulfide bonds in both WSSV proteins, suggesting that PmGILT exerts its anti-WSSV activity via its thiol reductase activity to disrupt the WSSV protein complex and restore the melanization activity of PmproPO1 and PmproPO2.
\end{abstract}

KEY WORDS: PmGILT · Yeast 2-hybrid · In vitro pull-down assay $\cdot$ WSSV protein $\cdot$ Disulfide bond reduction

\section{INTRODUCTION}

White spot syndrome virus (WSSV) is an enveloped DNA virus that infects cultured shrimp and many other crustaceans. It has a huge impact on shrimp farming, in which it causes large economic losses, with the cumulative mortality of WSSV-infected shrimps reach-

\footnotetext{
*Corresponding author: pamornra@yahoo.com
}

ing as high as $100 \%$ within 3 to $10 \mathrm{~d}$. Researchers have attempted to find ways to prevent WSSV infections. To that end, methods by which WSSV infects and replicates within hosts, the morphology of WSSV virions, and the characteristics of shrimp immune responses after WSSV infection have been intensively investigated (Verbruggen et al. 2016).

(C) The authors 2019. Open Access under Creative Commons by Attribution Licence. Use, distribution and reproduction are unrestricted. Authors and original publication must be credited. 
WSSV structural proteins are organized into 3 layers, including a nucleocapsid, a tegument, and an outer envelope that surrounds its DNA genome. In addition, the structural proteins of WSSV interact with each other to form complexes (Zhou et al. 2009, Chang et al. 2010, Huang et al. 2014, Liu et al. 2014), and new WSSV structural protein complexes are continually being reported (Huang et al. 2014).

In vertebrates, gamma-interferon ( $\gamma$-IFN)-inducible lysosomal thiol reductase (GILT), an endosomal/lysosomal-localized protein, is a key molecule in the adaptive immune system. GILT is constitutively expressed and participates upstream of major histocompatibility complex (MHC) class I- and class IIrestricted antigen presentation pathways in antigenpresenting cells (APCs). In the antigen presentation pathway, GILT reduces the intrachain disulfide bonds of cysteine-containing antigens. The reduced antigens are subsequently cleaved by various proteases, after which they form complexes with MHC proteins and translocate to the cell surface. There, they mount $\mathrm{CD}^{+} \mathrm{T}$ cell responses (Rocha \& Neefjes 2008) and prime naïve $\mathrm{CD}^{+}$cells to eliminate viruses, viral-derived antigens (Sealy et al. 2008, Singh \& Cresswell 2010) and tumors (Haque et al. 2002). In addition to its role in presentation pathways, GILT has been reported to act as a $\gamma$-IFNstimulated antiviral molecule that directly restricts the early and late phases of retrovirus infection by reducing the intrachain disulfide bonds of viral envelope proteins and proteins involved in viral propagation (Teramoto et al. 2013, Kubo et al. 2016)

In crustaceans, GILT was first reported in Penaeus monodon, in which it was named PmGILT. PmGILT is involved in the shrimp innate immune response, and its expression is up-regulated after injection with LPS and WSSV (Kongton et al. 2011). PmGILT also has thiol reductase activity, as has been shown in vertebrates (Nualla-ong et al. 2017). Because inverte- brate organisms lack antigen presentation activities, it is believed that in invertebrate organisms, GILT functions as an effector immune molecule which fights infectious microorganisms directly. Nualla-ong et al. (2017) revealed that PmGILT neutralized the entry of WSSV into shrimp cells both in vitro and in vivo. However, the anti-WSSV mechanism by which PmGILT performs this function was not defined. Therefore, the current study was performed using a yeast 2-hybrid assay ( $\mathrm{Y} 2 \mathrm{H}$ ) to identify the WSSV proteins targeted by PmGILT and to determine whether the targeted proteins have intrachain disulfide bonds that are reduced by PmGILT. The results of this study contribute to our understanding of shrimp innate immune responses and promote our ability to protect shrimp against WSSV infections.

\section{MATERIALS AND METHODS}

\section{1. pGBKT-7-PmGILT construction}

The BD-PmGILT bait vector was constructed by cloning the open reading frame of PmGILT in-frame into the pGBKT-7 vector, using specific forward and reverse primers containing Eco RI and BamHI recognition sites, respectively (Table 1). The recombinant plasmid was verified for a correct in-frame sequence by nucleotide sequencing. Before using the BDPmGILT plasmid in the $\mathrm{Y} 2 \mathrm{H}$, the plasmid was first tested for autoactivation.

\subsection{Yeast 2-hybrid (Y2H) assay}

To identify the WSSV proteins that interact with PmGILT, Y2H screening was performed using the Matchmaker ${ }^{\circledR}$ Gold Yeast Two-hybrid system (Clontech Laboratories) according to the manufacturer's

Table 1. Primers used in this experiment. Restriction sites (for ECoRI, BamHI, and XhoI, respectively) are $\underline{\text { underlined }}$

\begin{tabular}{|c|c|c|c|}
\hline Primer & Sequence $\left(5^{\prime}-3^{\prime}\right)$ & Amplicon size (bp) & Annealing temp. $\left({ }^{\circ} \mathrm{C}\right)$ \\
\hline BD-PmGILT-F & GAA TTC ATG CGT ACG TCT CTC CTT & 669 & 52 \\
\hline BD-PmGILT-R & $\overline{\text { GGA TCC }}$ TTA GGC AAA AGT GAC GGC & & \\
\hline WSSV002-BamF & $\overline{\text { GGA TCC }}$ ATG ACT GTT CCA GAA ATG G & 198 & 52 \\
\hline WSSV002-XhoR & CTC GAG CTA AAC TGG AGT GAG CTC AA & & \\
\hline WSSV164-BamF & GGA TCC ATG TCT CAC ATC AAC TCT AC & 1233 & 50 \\
\hline WSSV164-XhoR & CTC GAG TTA CTG GTA TCT GAT TTC TTG & & \\
\hline WSSV189-BamF & $\underline{\text { GGA TCC }}$ ATG GAA TGG ATA AAC CAA CGG & 692 & 55 \\
\hline WSSV189-XhoR & CTC GAG TTA TTG GAT AAA GTA GTT TA & & \\
\hline WSSV471-BamF & GGA TCC ATG GAG GAC CTA AAA TCC AC & 492 & 55 \\
\hline WSSV471-XhoR & CTC GAG TTA TGC ATT GTT TGT ATA CAC C & & \\
\hline
\end{tabular}


protocol. Briefly, the BD-PmGILT plasmids were transformed into the yeast strain Y2HGold to produce a Gal4 DNA-binding domain fusion PmGILT. Activation domain (AD)-WSSV library plasmids (generously provided by Dr. Sangchan Senapin, NSTDA, Thailand) were transformed into the yeast strain Y187 and expressed Gal4 activation domain fusion WSSV proteins. Then, to screen the candidate WSSV proteins for interactions with PmGILT, both yeast strains were co-cultured to create diploid cells. To detect diploid cells that exhibited protein-protein interactions, the diploid cells were spread on a synthetic defined (SD) medium that lacked leucine and tryptophan (SD/-Leu/-Trp) but contained X-alphaGal (DDO/X). To confirm the protein-protein interactions, the blue colonies that grew on the DDO/X medium were subsequently plated onto a higher stringency, quadruple-dropout medium that lacked leucine, tryptophan, adenine and histidine (SD/ -Leu/-Trp/-Ade/-His) but contained X-alpha-Gal and Aureobasidin A (QDO/X/A). The plasmids were then isolated from positive clones that grew on QDO/X/A medium, and the quality of inserts was checked by PCR using pGADT-7-specific primers. The isolated yeast plasmids were transformed into Escherichia coli top 10F' cells and screened on LuriaBertani (LB) agar containing ampicillin (final concentration, $100 \mathrm{\mu g} \mathrm{ml}^{-1}$ ). Finally, the AD-WSSV plasmids in the bacterial colonies were isolated and subjected to DNA sequencing. To confirm the screening results, the Y2H gold strains were co-transformed with BD-PmGILT, and the candidate AD-WSSV plasmids were plated onto DDO/X and QDO/X/A medium according to the manufacturer's protocols.

\subsection{Domain searches, 3-dimensional (3D) modeling and docking analysis}

The motif and domains present in PmGILT-interacting WSSV proteins were searched using the PROSITE and SMART programs using the default settings. A molecular docking simulation was performed to illustrate the interaction complexes of PmGILT and WSSV proteins, including PmGILT/WSSV164 and PmGILT/WSSV189, using the ClusPro 2.0 server (http://cluspro.bu.edu). Because there are no 3D structures of PmGILT and WSSV proteins available in the Protein Data Bank, the threading modeling approach with the I-TASSER server (Zhang 2008, Roy et al. 2010, Yang et al. 2015) and homology modeling with SWISS-MODEL server (Arnold et al. 2006, Bordoli et al. 2009, Biasini et al. 2014) were combined to generate 3D coordinate files of all proteins for the docking simulation. The top-ranking model of docked results with the lowest docking energy value of the ClusPro 2.0 server was delegated to analyze the interaction in detail. Protein-protein docking was performed using the ClusPro 2.0 server (Comeau et al. 2004, Kozakov et al. 2017). The 3D models and the surfaces at interaction sites were visualized using the PyMOL program.

\subsection{Expression and purification of recombinant glutathione-S-transferase (GST)-fusion rWSSV proteins}

The full coding sequences of the WSSV genes were cloned into pGEX-4T-1 using specific PCR primers, which were flanked at the ends of the forward and reverse primers with BamHI and XhoI restriction sequences, respectively (Table 1 ). Here, these recombinant plasmids are called pGEX-WSSVs. After sequence verification, the pGEX-WSSV plasmids were transformed into the E. coli strain BL21 expression host to produce GST-rWSSV proteins. To efficiently achieve a soluble yield of GST-rWSSV proteins, the transformants were cultured in LB broth containing ampicillin $\left(100 \mu \mathrm{g} \mathrm{ml}^{-1}\right)$ and incubated at $37^{\circ} \mathrm{C}$ with shaking until an $\mathrm{OD}_{600}$ of 0.5 was achieved. The transformant was then induced by adding isopropyl $\beta$-D-1 thiogalactopyranoside (IPTG) at a final concentration of $0.1 \mathrm{mM}$ and incubation at $28^{\circ} \mathrm{C}$ at a shaking speed of $180 \mathrm{rpm}$ for $3 \mathrm{~h}$. The GST-rWSSV proteins were then extracted from the bacterial cells using GST extraction buffer and sonication. The supernatant fraction containing the GST-rWSSV proteins was analyzed by SDS-PAGE, and proteins were detected by Western blot analysis using an anti-GST antibody.

\subsection{Expression and purification of recombinant His-fusion proteins}

Cultured E. coli BL 21 (DE3) cells carrying pETPmGILT/WSSVs recombinant plasmids were incubated in LB broth containing $50 \mathrm{\mu g} \mathrm{ml}^{-1}$ of kanamycin until the $\mathrm{OD}_{600}$ was approximately 0.4 to 0.6 . Induction was then performed by adding IPTG at a final concentration of $0.5 \mathrm{mM}$, after which the cells were cultured at $37^{\circ} \mathrm{C}$ at a shaking speed of $180 \mathrm{rpm}$ for $4 \mathrm{~h}$. The resulting His-fusion recombinant proteins were insoluble. Before purification, the inclusion bodies were dissolved according to the protocol described 
by Favacho et al. (2006). Briefly, the inclusion bodycontaining pellet was solubilized in denaturing buffer B (0.01 M Tris-HCl, 0.1 M sodium phosphate buffer, and $8 \mathrm{M}$ urea, pH 8.0) and incubated on ice for $1 \mathrm{~h}$. Then, the supernatant was collected after centrifugation at $14180 \times g$ for $10 \mathrm{~min}$. The His-fusion proteins were purified using Pierce Ni-NTA Superflow agarose (Thermo Fisher) according to the manufacturer's protocol.

The purified His-fusion proteins were dialyzed in Tris-buffered saline containing decreasing amounts of urea $(6,4,2$ and $1 \mathrm{M})$ at $4^{\circ} \mathrm{C}$. The dialysis procedure was repeated twice. For His-rPmGILT, a final dialysis against acetate buffer, pH 5.5 (100 mM sodium chloride, $50 \mathrm{mM}$ sodium acetate $\mathrm{pH}$ 5.5, and $0.1 \%$ Triton-X-100), was also performed. The proteins were examined by SDS-PAGE and Western blot analysis using an anti-His-tagged antibody.

\subsection{In vitro pull-down assay}

To confirm the interactions between GST-rWSSVs and His-rPmGILT, a GST-pull-down assay was used. First, the rGST (control) or GST-rWSSV proteins were embedded in Glutathione Superflow agarose for $2 \mathrm{~h}$ at $4^{\circ} \mathrm{C}$ with gentle rocking. Then, the beads were washed and incubated with purified His-rPmGILT for another $2 \mathrm{~h}$ at $4^{\circ} \mathrm{C}$ with gentle rocking. After the incubation, the beads were washed. Then, $5 \times$ loading dye was added to the beads, and they were boiled for $5 \mathrm{~min}$. The protein complexes in the solution were analyzed by Western blotting using an anti-His antibody.

\subsection{Disulfide bond reduction assay}

To determine whether cysteine-containing WSSV proteins are a substrate of PmGILT, a disulfide bond reduction assay followed by a thiol reductase assay was performed according to Lackman et al.'s (2007) protocol. A thiol-free cysteine detection assay was then performed using the protocol of Kubo et al. (2016) with some modifications. Biotin-maleimide (Sigma-Aldrich, USA) was used to detect any proteins containing free thiol groups. Briefly, purified His-rPmGILT (50 $\mu \mathrm{g} \mathrm{ml} \mathrm{m}^{-1}$ ) was preactivated with $20 \mu \mathrm{M}$ dithiothreitol (DTT) for $10 \mathrm{~min}$ at room temperature. Then, the preactivated rPmGILT was added to purified His-rWSSV164 and/or HisrWSSV189 $\left(50 \mu \mathrm{g} \mathrm{ml}^{-1}\right)$, and the mixture was incubated for $1 \mathrm{~h}$ with agitation. After incubation, the thiol group-containing proteins were incubated with biotin-maleimide (final concentration: $500 \mu \mathrm{g} \mathrm{ml} \mathrm{m}^{-1}$ ) for $1 \mathrm{~h}$ with agitation. The biotinylated proteins were isolated by incubating them for $1 \mathrm{~h}$ with avidinagarose. The biotinylated proteins were then subjected to SDS-PAGE and Western blot analysis.

\section{RESULTS}

\subsection{Identification of the WSSV target protein of PmGILT}

Based on the neutralizing activity exerted by PmGILT against WSSV infection (Nualla-ong et al. 2017), we hypothesized that PmGILT might interact with WSSV structural proteins to restrain WSSV infectivity. PmGILT-interacting WSSV proteins were identified by $\mathrm{Y} 2 \mathrm{H}$. The results revealed 10 candidate clones that were able to grow and activate the reporter genes, resulting in blue colonies on QDO/ X/A medium. When the candidate clones were sequenced, a BLAST search of the sequences was performed, and all 10 clones matched WSSV002 (GenBank acc. no. AAL88870.1), WSSV164 (acc. no. AAL89032.1), WSSV189 (acc. no. AAL89057.1) or WSSV471 (acc. no. AAL89339.1) with similarities of 99 to $100 \%$. The confirmation of these positive interactions and the details for each WSSV protein are shown in Fig. 1 and Table 2, respectively. While the information available on these WSSV genes is limited, WSSV189 and WSSV471 are reported to be structural proteins (Suraprasit et al. 2014, Methatham et al. 2017), whereas WSSV164 is an immediate-early (IE) protein (Li et al. 2009), and WSSV002 is an unknown protein.

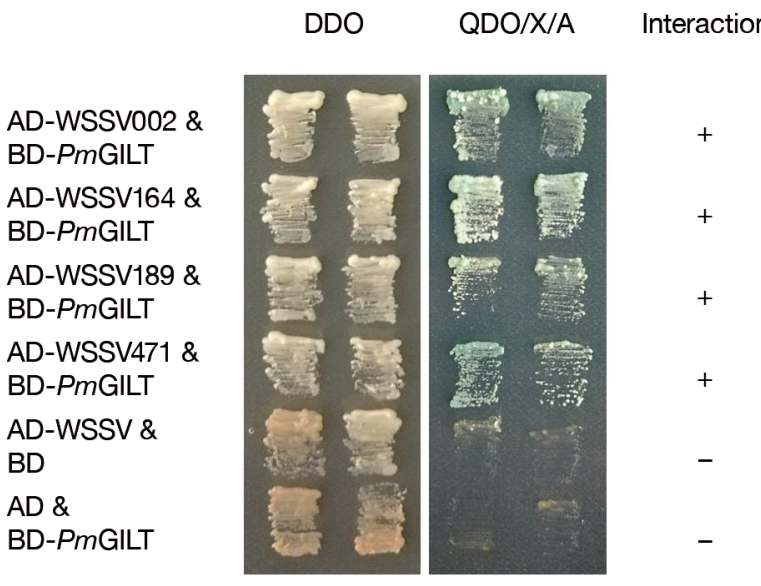

Fig. 1. Verification of the interactions between PmGILT and white spot syndrome virus (WSSV) proteins. In these experiments, the BD-PmGILT and AD-WSSV ORF plasmids were co-transformed into shared host Y2HGold cells 
Table 2. Summary information for the PmGILT-interacting white spot syndrome virus (WSSV) proteins identified in this study

\begin{tabular}{|c|c|c|c|c|c|c|}
\hline \multirow[t]{2}{*}{ Name } & \multicolumn{2}{|c|}{$\_$Size $\_$} & \multirow{2}{*}{$\begin{array}{c}\text { Sequence } \\
\text { identity (\%) }\end{array}$} & \multirow{2}{*}{$\begin{array}{c}\text { Position } \\
\text { within virion }\end{array}$} & \multirow{2}{*}{$\begin{array}{c}\text { Temporal } \\
\text { analysis }\end{array}$} & \multirow{2}{*}{$\begin{array}{l}\text { Cysteine } \\
\text { position(s) }\end{array}$} \\
\hline & (bp) & (aa) & & & & \\
\hline WSSV002 & 186 & 61 & 100 & Unknown & - & C39 \\
\hline \multirow[t]{4}{*}{ WSSV164 } & 1221 & 406 & 99 & Unknown & $\begin{array}{c}\text { Immediate- } \\
\text { Early }^{\mathrm{a}}\end{array}$ & $\mathrm{C} 20$ \\
\hline & & & & & & $\mathrm{C} 226$ \\
\hline & & & & & & C283 \\
\hline & & & & & & C315 \\
\hline \multirow[t]{3}{*}{ WSSV189 } & 669 & 222 & 100 & Envelope $^{\mathrm{b}}$ & Late $^{b}$ & C137 \\
\hline & & & & & & C150 \\
\hline & & & & & & $\mathrm{C} 172$ \\
\hline WSSV471 & 480 & 159 & 100 & Envelope $^{\mathrm{C}}$ & Late $^{b}$ & - \\
\hline
\end{tabular}

74, and $55 \mathrm{kDa}$ corresponded to GST-rWSSV002 (Fig. 2a), GSTrWSSV164 (Fig. 2b), and GSTrWSSV189 (Fig. 2c), respectively. Likewise, His-fusion proteins were produced using Escherichia coli strain BL21(DE3). The sizes of the His-rPmGILT, His-rWSSV164, and His-rWSSV189 proteins were 27,48 , and $29 \mathrm{kDa}$, respectively (Fig. 3). Western blotting using an anti-His antibody indicated that the bands at 27, 48, and $29 \mathrm{kDa}$ corresponded to HisrPmGILT (Fig. 3a), His-rWSSV164 (Fig. 3b), and His-rWSSV189 (Fig. 3c), respectively.

\subsection{Domain searches for PmGILT-interacting WSSV proteins}

The crucial domains present in PmGILT-interacting WSSV proteins were investigated using the PROSITE and SMART programs. The results indicate that WSSV471 contains a coiled-coil domain, while WSSV002, WSSV164 and WSSV189 had no conserved domains or motifs. Even so, they had low complexity and contained $\mathrm{N}$-glycosylation sites, casein kinase II phosphorylation sites, and protein kinase C phosphorylation sites. In addition, WSSV002, WSSV189 and WSSV164 also contained $N$-myristoylation sites. Moreover, WSSV164 and WSSV471 each possessed a tyrosine kinase phosphorylation site (Table A1 in the Appendix). However, the exact role of these WSSV proteins in WSSV infection remains unclear, except for WSSV164, which has been reported to interfere with the shrimp melanization cascade (Sangsuriya et al. 2018).

\subsection{WSSVs and PmGILT protein production}

The GST-fusion rWSSV proteins were produced by a prokaryotic expression system using Escherichia coli strain BL21. Three of the 4 WSSV proteins - WSSV002, WSSV164 and WSSV189-were successfully produced (Fig. 2). The GST-fusion rWSSV proteins were detected and found to have the expected sizes (including $29 \mathrm{kDa}$ of GST) of approximately 36,74 , and $55 \mathrm{kDa}$, respectively. Western blotting performed using an anti-GST antibody confirmed that the bands corresponding to 36 ,

\subsection{In vitro protein-protein interactions in GST- pull-down assays}

The crude cell lysates of GST-fusion WSSV proteins and purified His-rPmGILT were used to perform GST-pull-down assays. The results confirmed the interactions between His-PmGILT and GST-rWSSV proteins because both proteins were detected, whereas the rGST protein did not bind with the HisrPmGILT protein (Fig. 4). These results indicate that PmGILT interacts with WSSV proteins to support its neutralizing activity.

\subsection{PmGILT reduced the intrachain disulfide bonds of WSSV proteins}

We next investigated the antiviral mechanism by which GILT exerts its thiol reductase activity to break down the intrachain disulfide bonds of cysteine-containing proteins, thereby inhibiting viral envelope protein-mediated infection and restraining viral propagation in cells (Kubo et al. 2016). PmGILT contains one cysteine in its active site but still has thiol reductase activity (Nualla-ong et al. 2017). Thus, it was assumed that the anti-WSSV activity of PmGILT could involve its thiol reductase activity, similar to what was shown for human GILT. In disulfide bond reduction assays, biotinylated rWSSV proteins were reproducibly increased by rPmGILT as well as by DTT (positive control) (Fig. 5), indicating that PmGILT had digested the intrachain disulfide bonds of the WSSV proteins. Overall, these data indicate that PmGILT interacts with the WSSV proteins to digest their intra- 
a

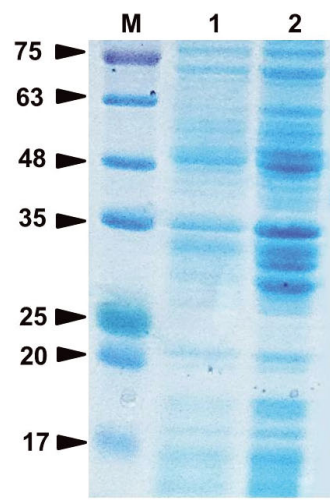

C

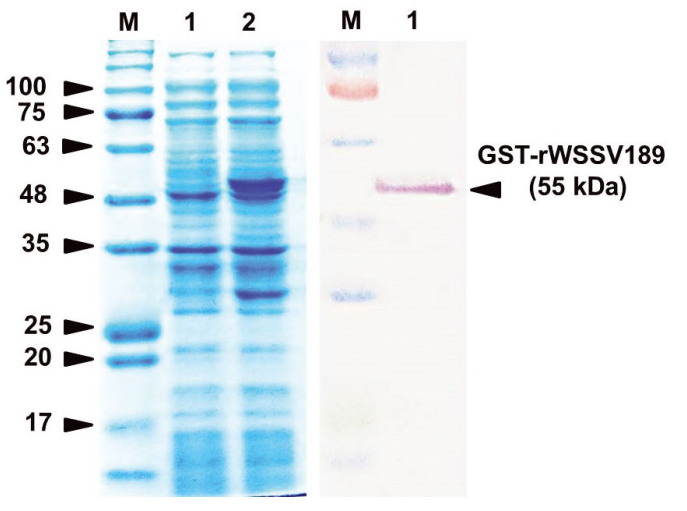

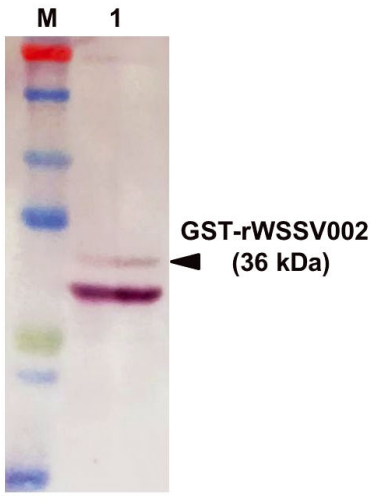

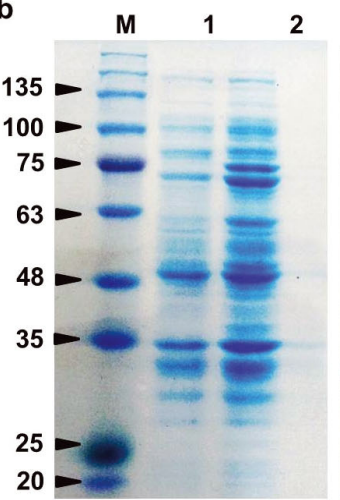

M 1

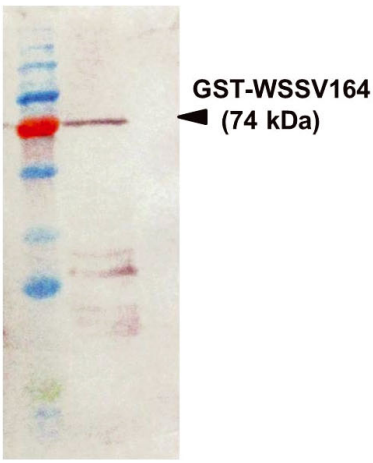

Fig. 2. Expression (left) and Western blot analysis (right) of (a) recombinant GST-WSSV002, (b) WSSV164, and (c) WSSV189 in Escherichia coli. Recombinant GST-rWSSV expression was analyzed by $10 \%$ SDS-PAGE. Lane $M$ contains a protein marker, Lane 1 contains non-induced cell lysate, and Lane 2 contains induced cell lysate. The supernatant fraction of the bacterial cell lysates was subjected to Western blot analysis using a GST-specific antibody
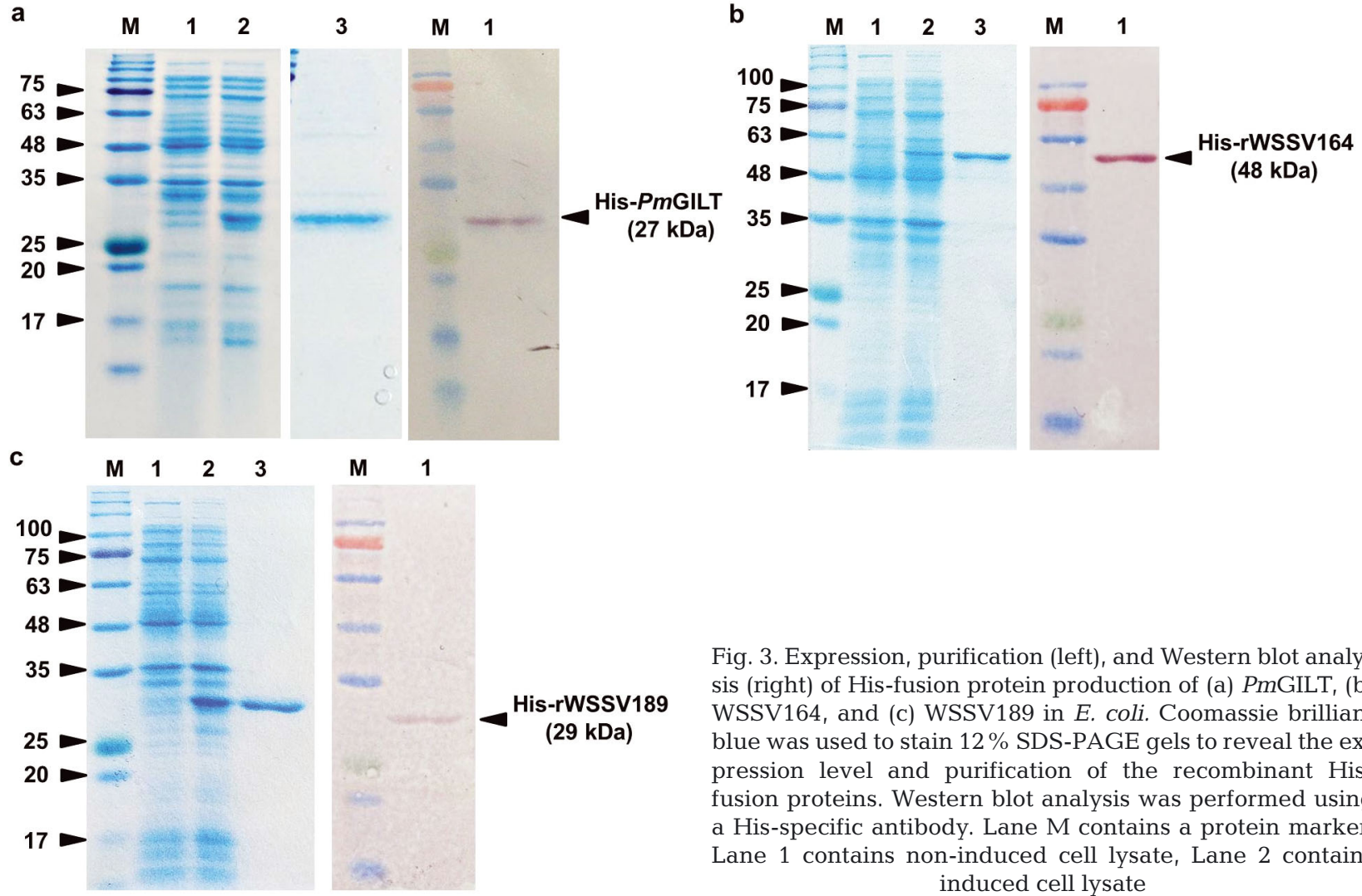

Fig. 3. Expression, purification (left), and Western blot analysis (right) of His-fusion protein production of (a) PmGILT, (b) WSSV164, and (c) WSSV189 in E. coli. Coomassie brilliant blue was used to stain $12 \%$ SDS-PAGE gels to reveal the expression level and purification of the recombinant Hisfusion proteins. Western blot analysis was performed using a His-specific antibody. Lane $\mathrm{M}$ contains a protein marker, Lane 1 contains non-induced cell lysate, Lane 2 contains induced cell lysate 


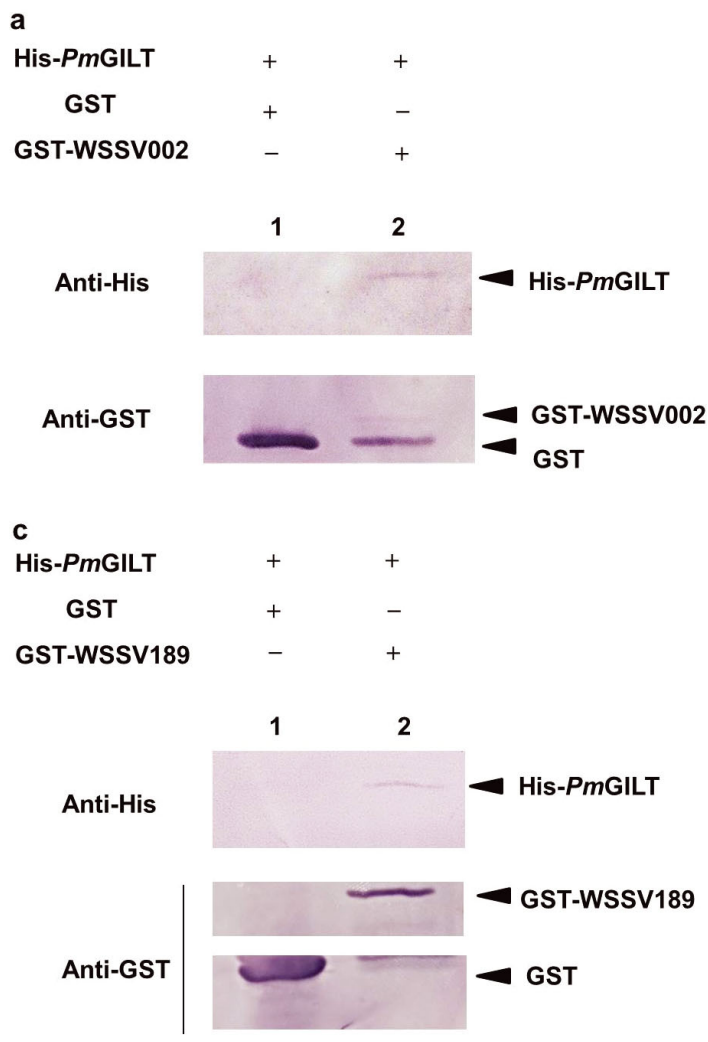

chain disulfide bonds. This interaction represents a potential mechanism for the anti-WSSV activity of PmGILT.

\subsection{Predicted 3D structures and protein-protein interactions}

From the results above, PmGILT could interact with WSSV proteins and also reduce the intrachain disulfide bonds within WSSV164 and WSSV189. Subsequently, to describe the details of their interaction and the mechanism of PmGILT, a protein-protein docking simulation was performed.

A PmGILT predicted model (Fig. 6a) was assigned as a receptor protein and WSSV164 (Fig. 6b) and WSSV189 (Fig. 6c) as ligand proteins. The top-ranking interaction complex based on the center and lowest binding energy score was selected for details of the binding sites. The analyzed results demonstrated that PmGILT/WSSV164 showed a higher binding energy score of interaction than PmGILT/WSSV189. The center binding energy and lowest binding energy of PmGILT/WSSV164 were $-1133.70 \mathrm{Kcal}$ $\mathrm{mol}^{-1}$ and $-1224.80 \mathrm{Kcal} \mathrm{mol}^{-1}$, respectively,

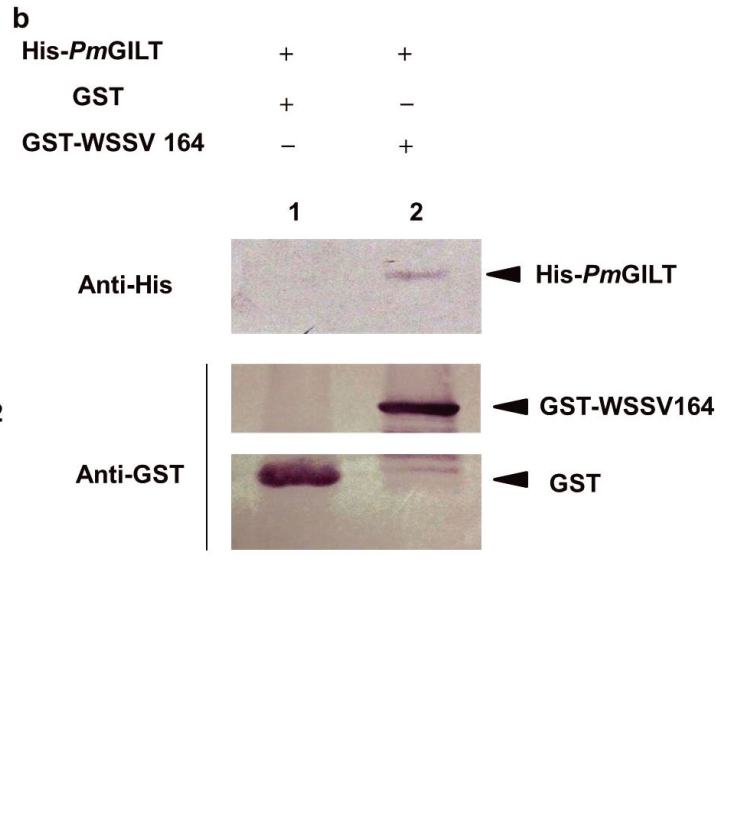

Fig. 4. In vitro protein-protein interactions were evaluated using glutathione-S-transferase (GST)-pull-down assays, performed with crude GST-expressing E. coli cell lysate (Lane 1) or GST-rWSSV proteins (Lane 2) (a) WSSV002, (b) WSSV164, and (c) WSSV189 using purified His-PmGILT

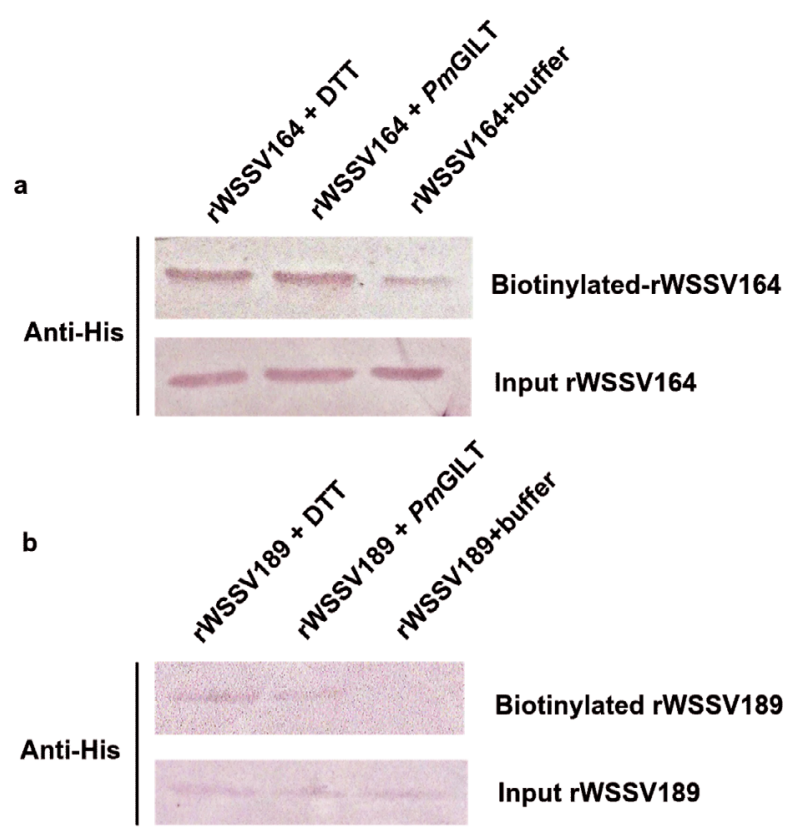

Fig. 5. In vitro disulfide bond reduction assays. Biotinylated (a) WSSV164 and (b) WSSV189 levels increased after coincubation with DTT (positive control, first lane) and HisrPmGILT (middle lane) relative to the levels induced by the buffer treated without DTT or PmGILT (last lane) 
whereas those of the PmGILT/WSSV189 were $-718.90 \mathrm{Kcal} \mathrm{mol}^{-1}$ and $-799.30 \mathrm{Kcal} \mathrm{mol}^{-1}$, respectively.

The binding region analysis of the PmGILT/ WSSV164 interaction complex revealed that the Nterminal of PmGILT (residues 1-90) bound to the C-terminal of WSSV164 (residues 300-405) (Fig. 6d). In addition, residues 1-90 of PmGILT were found to interact with 3 regions of WSSV189 at residues 80-110, 150-165, and 205-222, respectively (Fig. 6e).
These results showed that the interaction of PmGILT and WSSV proteins occurred on PmGILT's active site-containing region and the cysteine-containing regions of WSSV proteins.

\section{DISCUSSION}

Antigen presentation is a mechanism that occurs in the adaptive immune system of vertebrates. After

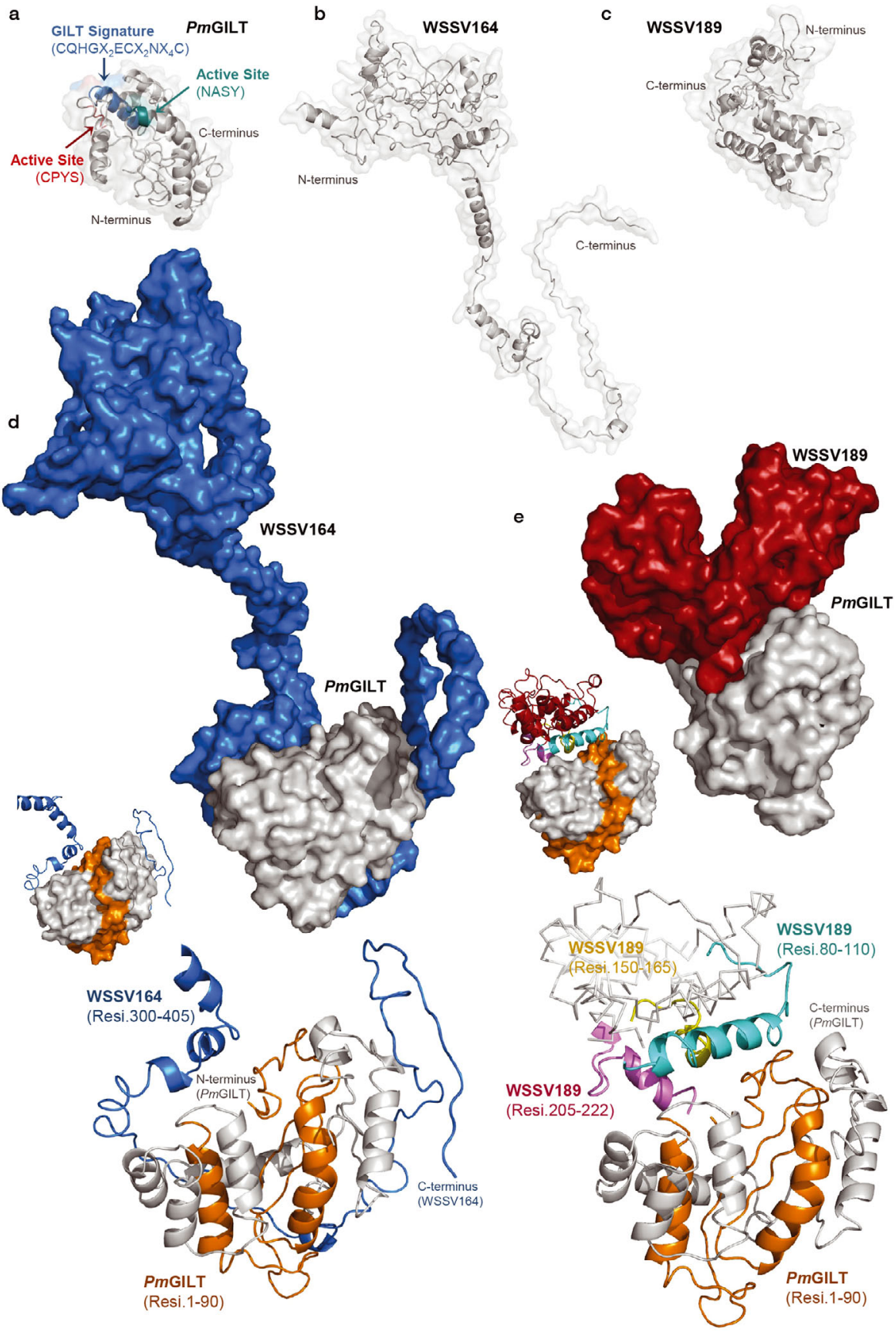

Fig. 6. PmGILT and WSSV protein interaction complexes. 3D models of (a) PmGILT, (b) WSSV164, and (c) WSSV189 are shown. The interaction complex of PmGILT and WSSV proteins was simulated by the ClusPro 2.0 server. The top-ranking binding energy complex of interaction was selected for identification of binding regions. (d) The binding residues of PmGILT/WSSV164. The analysis found that residues 1-90 (orange) of PmGILT facilitate binding to WSSV164 at residues 300-405 (navy blue). (e) Three regions of WSSV189 residues 80-110 (turquoise), 150-165 (yellow), and 205-222 (cherry red) are shown with the interaction sites with PmGILT residues 1-90 (orange) 
protein antigens are internalized by endocytosis and/ or phagocytosis, GILT facilitates this pathway by reducing the disulfide bond of cysteine-containing antigens to generate unfolded antigens that are further degraded by proteases. The antigenic peptides are then bound with MHC class I or class II molecules to form MHC-peptide complexes. Finally, these complexes translocate to the cell surface of antigen-presenting cells (APCs), and T cells are then stimulated to eliminate the infected cells (Sinnathamby et al. 2004, Sealy et al. 2008, Singh \& Cresswell 2010).

In addition to APC cells, GILT also acts directly as a $\gamma$-IFN-stimulated antiviral molecule to combat infection by various retroviruses. These activities occur via its thiol reductase activity, which inhibits envelope protein-mediated infection and viral progeny production (Teramoto et al. 2013, Kubo et al. 2016).

Antigen presentation is not reported in invertebrate organisms. However, GILTs have been cloned and characterized from echinoderms (Ren et al. 2015), crustaceans (Kongton et al. 2011, Huang et al. 2015), insects (Kongton et al. 2014), and mollusks (De Zoysa \& Lee 2007, Zhang et al. 2010) and play key roles in the innate immune response. Invertebrate GILTs are upregulated in immune and digestive organs after bacterial infection (De Zoysa \& Lee 2007, Zhang et al. 2010, Kongton et al. 2011, 2014, Huang et al. 2015, Ren et al. 2015). The over-expression of Drosophila GILT in immune organs led to reduced numbers of bacteria in the same organs (Kongton et al. 2014).

Some invertebrate GILTs also respond to viral infection (Kongton et al. 2011, Ren et al. 2015). In sea cucumber, the expression of GILT was up-regulated in coelomocytes after treatment with poly (I:C) (a synthetic double-stranded RNA). The promoter region of sea cucumber GILT contains a nuclear factor $\kappa$-lightchain-enhancer of activated B cells (NF- $\mathrm{kB}$ ) binding site and $\gamma$-IFN activation site. It is suggested that sea cucumber GILT may be involved in the immune response against viral infection via both the Janus kinase/signal transducers of transcription and activators of transcription and Toll-like receptor3-mediated NF-KB signaling pathways (Ren et al. 2015).

In crustaceans, GILT was also cloned and characterized in black tiger shrimp, namely, PmGILT. The expression of PmGILT was induced after injection with LPS and WSSV solution (Kongton et al. 2011). PmGILT reduced WSSV infectivity when recombinant PmGILT was incubated with WSSV (Nualla-ong et al. 2017). However, the details of the anti-WSSV mechanism involved in this process remain unclear. Based on what is known about the neutralizing activity of PmGILT, we hypothesized that the effect of
PmGILT on anti-WSSV activity might be through interactions with WSSV structural proteins through interference with viral infection and/or replication or reducing their effects the melanization pathway. PmGILT interacted with WSSV proteins WSSV002, WSSV164, and WSSV189, in both Y2H assays (Fig. 1) and GST-pull down assays (Fig. 4). WSSV189 and WSSV471 are structural proteins and are located in the envelope layer (Suraprasit et al. 2014, Methatham et al. 2017), whereas WSSV164 is an IE protein (Li et al. 2009). No report previously described WSSV002. A domain search performed using the PROSITE and SMART programs revealed that WSSV164, WSSV189 and WSSV471 have multiple $N$-glycosylation sites (Table A1). It is possible that PmGILT-mediated reduction strongly affects viral proteins, similar to what has been observed for the human GILT protein (Singh \& Cresswell 2010).

The thiol reductase activity of GILT is essential for its function. In vertebrates, GILT contains 2 cysteines in its active site (a $\mathrm{C}_{46} \mathrm{XXC}_{49}$ motif). Usually, both cysteines at the GILT active site cooperate to reduce disulfide bonds in target proteins. The N-terminal cysteine (Cys-46) initially attacks the disulfide bond of the substrate, forming a mixed disulfide GILT-substrate complex. Subsequently, an intrachain disulfide bond is formed between Cys-46 and Cys-49 of GILT, and the reduced substrate is then released. Despite the presence of a mutation at its $\mathrm{C}$-terminal cysteine (Cys-49), GILT was still able to reduce the disulfide bonds of its substrate, but at lower levels, and GILT was subsequently trapped with the substrate (Phan et al. 2000).

The active site of PmGILT in shrimp is different from that of GILT proteins in vertebrates, and the active site of the shrimp PmGILT contains only an Nterminal cysteine (Cys-32) (the active site of PmGILT is a CXXS motif) (Kongton et al. 2011). However, PmGILT still has thiol reductase activity (Nualla-ong et al. 2017). Given PmGILT's thiol reductase activity, our next question was whether its anti-WSSV activity involves its thiol reductase activity. If so, cysteinecontaining WSSV proteins might be reduced at their intrachain disulfide bonds. Among the identified PmGILT-interacting WSSV proteins, WSSV164 and WSSV189 contained 4 and 3 cysteines, respectively, while WSSV002 contained only 1 cysteine (Table 2 ). Thus, WSSV164 and WSSV189 were subjected to disulfide bond reduction assays. These assays revealed that the intrachain disulfide bonds of these 2 selected WSSV proteins were reduced by PmGILT (Fig. 5). Similarly, Wunderlich et al. (1995) reported that DbsA, a protein disulfide isomerase in E. coli, 
contained 1 cysteine at the $\mathrm{N}$-terminus in its active site and was capable of catalyzing disulfide-interchange reactions as well as the wild-type, resulting in unfolded substrate. Schleicher et al. (2018) revealed that mouse GILT with a mutated active site (C2, CxxS) still exhibited the reductase activity in vitro. In the present study, protein docking predictions revealed that the active site of PmGILT was rotated to face the cysteine-containing region of WSSV proteins. It is therefore possible that PmGILT with a cysteine at only the N-terminal site could still have thiol reductase activity and could therefore unfold its substrate, especially disulfide bond-containing WSSV proteins. Based on our results, we propose 2 mechanisms by which PmGILT activity against WSSV infection may occur.

From the WSSV proteins interaction map data reported by Sangsuriya et al. (2014), WSSV189 and WSSV471 were shown to form a viral envelope protein complex along with another 3 WSSV structural proteins, including WSSV395 (envelope protein), WSSV458 (tegument protein), and WSSV186 (nucleocapsid protein) (Suraprasit et al. 2014, Jaree et al. 2016, Methatham et al. 2017). It is possible that WSSV enters cells through the endocytosis pathway, and GILT reduces the intrachain disulfide bonds of the WSSV target proteins within endosomal/endolysosomal compartments, resulting in destruction of the structural protein complex of incoming WSSV and subsequent interference with viral infection and/or replication.

Alternatively, it is possible that PmGILT may be involved in the restoration of the melanization cascade in shrimp during WSSV and other viral infections. IE proteins are crucial for the life cycle and the expression of downstream early and late genes of viruses (Li et al. 2009). Additionally, IE proteins can alter the functions of host genes and regulate the host immune response (Buisson et al. 1989, Kenney et al. 1989, Holley-Guthrie et al. 1990). WSSV164 interferes with the shrimp melanization cascade through binding to PmproPO1 and PmproPO2 proteins (Sangsuriya et al. 2018). In the present study, we showed that PmGILT can bind to and reduce the disulfide bond of WSSV164, resulting in the separation of the denatured WSSV164 from the WSSV164-PmproPO complex. Subsequently, the free PmproPOs could be converted into active PmPOs by proPO-activating enzymes. The active PmPOs could be activated in melanin production (Tassanakajon et al. 2013). The eradication of the WSSV164-PmproPO complex by PmGILT needs to be further investigated.

\section{CONCLUSION}

These observations support the following hypothetical scheme by which PmGILT could exert its antiWSSV activities. PmGILT binds to WSSV proteins to reduce their intrachain disulfide bonds or the interchain disulfide bonds between WSSV proteins. Eventually, the WSSV target proteins become denatured, and this affects the development of WSSV protein complexes and interactions with components of the immune system.

Acknowledgements. We thank the Royal Thai Government Scholarship supported by the Ministry of Science and Technology (THAILAND) and the Royal Golden Jubilee Graduate Program from the Thailand Research Fund (TRF) for support through a grant funded to J.T. and H.S. (4.J.PS/53/A.1), respectively. This work was also supported in part by Prince of Songkla University grants and TRG5780145 to T.P. The authors also thank Prof. Dr. Supa Hannongbua and Assist. Prof. Dr. Patchreenart Saparpakorn for their kind suggestions about protein-protein prediction. Finally, we thank the Publication Clinic, Research and Development Office, Prince of Songkla University, and Assoc. Prof. Seppo Karrila for their revision of the English.

\section{LITERATURE CITED}

Arnold K, Bordoli L, Kopp J, Schwede T (2006) The SWISSMODEL workspace: a web based environment for protein structure homology modeling. Bioinformatics 22: 195-201

*Biasini M, Bienert S, Waterhouse A, Arnold K and others (2014) SWISS MODEL: modelling protein tertiary and quaternary structure using evolutionary information. Nucleic Acids Res 42:252-258

Bordoli L, Kiefer F, Arnold K, Benkert P, Battey J, Schwede T (2009) Protein structure homology modelling using SWISS-MODEL workspace. Nat Protoc 4:1-13

* Buisson M, Manet E, Trescol-Biemont MC, Gruffat $\mathrm{H}$, Durand B, Sergeant A (1989) The Epstein-Barr virus (EBV) early protein EB2 is a posttranscriptional activator expressed under the control of EBV transcription factors EB1 and R. J Virol 63:5276-5284

Chang YS, Liu WJ, Lee CC, Chou TL and others (2010) 3D Model of the membrane protein complex formed by the white spot syndrome virus structural proteins. PLOS ONE 5:e10718

Comeau SR, Gatchell DW, Vajda S, Camacho CJ (2004) ClusPro: an automated docking and discrimination method for the prediction of protein complexes. Bioinformatics 20:45-50

*De Zoysa M, Lee J (2007) Molecular cloning and expression analysis of interferon- $\gamma$ inducible lysosomal thiol reductase (GILT)-like cDNA from disk abalone (Haliotis discus discus). J Invertebr Pathol 96:221-229

Favacho ARM, Kurtenbach E, Sardi SI, Gouvea VS (2006) Cloning, expression, and purification of recombinant bovine rotavirus hemagglutinin, VP8*, in Escherichia coli. Protein Expr Purif 46:196-203

*Haque MA, Li P, Jackson SK, Zarour HM and others (2002) 
Absence of $\gamma$-interferon-inducible lysosomal thiol reductase in melanomas disrupts $\mathrm{T}$ cell recognition of select immunodominant epitopes. J Exp Med 195:1267-1277

*Holley-Guthrie EA, Quinlivan EB, Mar EC, Kenney S (1990) The Epstein-Barr virus (EBV) BMRF1 promoter for early antigen (EA-D) is regulated by the EBV transactivators, BRLF1 and BZLF1, in a cell-specific manner. J Virol 64: 3753-3759

* Huang PY, Leu JH, Chen LL (2014) A newly identified protein complex that mediates white spot syndrome virus infection via chitin-binding protein. J Gen Virol 95: 1799-1808

Huang WS, Duan LP, Huang B, Zhou LH and others (2015) Identification of three IFN- $\gamma$ inducible lysosomal thiol reductase (GILT)-like genes in mud crab Scylla paramamosain with distinct gene organizations and patterns of expression. Gene 570:78-88

Jaree P, Senapin S, Hirono I, Lo CF, Tassanakajon A, Somboonwiwat K (2016) WSV399, a viral tegument protein, interacts with the shrimp protein PmVRP15 to facilitate viral trafficking and assembly. Dev Comp Immunol 59: 177-185

Kenney S, Holley-Guthrie E, Mar EC, Smith M (1989) The Epstein-Barr virus BMLF1 promoter contains an enhancer element that is responsive to the BZLF1 and BRLF1 transactivators. J Virol 63:3878-3883

Kongton K, Phongdara A, Tonganunt M, Wanna W (2011) Molecular cloning and expression analysis of the $\gamma$ interferon-inducible lysosomal thiol reductase gene from the shrimp Penaeus monodon. Mol Biol Rep 38: 3463-3470

Kongton K, Mccall K, Phongdara A (2014) Identification of gamma-interferon inducible lysosomal thiol reductase (GILT) homologues in the fruit fly Drosophila melanogaster. Dev Comp Immunol 44:389-396

Kozakov D, Hall DR, Xia B, Porter KA and others (2017) The ClusPro web server for protein-protein docking. Nat Protoc 12:255-278

Kubo Y, Izumida M, Yashima Y, Kamiyama YH and others (2016) Gamma-interferon-inducible, lysosome/endosome-localized thiolreductase, GILT, has anti-retroviral activity and its expression is counteracted by HIV-1. Oncotarget 7:71255-71273

* Lackman RL, Jamieson AM, Griffith JM, Geuze H, Cresswell P (2007) Innate immune recognition triggers secretion of lysosomal enzyme by macrophages. Traffic 8: 1179-1189

Ki F, Li M, Ke W, Ji Y, Bian X, Yan X (2009) Identification of the immediate-early genes of white spot syndrome virus. Virology 385:267-274

Liu WJ, Shiung HJ, Lo CF, Leu JH and others (2014) Characterization and interactome study of white spot syndrome virus envelope protein VP11. PLOS ONE 9: e85779

Methatham T, Boonchuen P, Jaree P, Tassanakajon A, Somboonwiwat K (2017) Antiviral action of the antimicrobial peptide ALFPm3 from Penaeus monodon against white spot syndrome virus. Dev Comp Immunol 69:23-32

Nualla-ong A, Saelim H, Kongton K, Phongdara A (2017) Biological activity of Penaeus monodon GILT in shrimp pathogen protection. Songklanakarin J Sci Technol 39: 261-268

Phan UT, Arunachalam B, Cresswell P (2000) Gamma-interferon inducible lysosomal thiol reductase (GILT). Maturation, activity, and mechanism of action. J Biol Chem
275:25907-25914

* Ren C, Chen T, Jiang X, Luo X, Wang Y, Hu C (2015) The first echinoderm gamma-interferon-inducible lysosomal thiol reductase (GILT) identified from sea cucumber (Stichopus monotuberculatus). Fish Shellfish Immunol 42: 41-49

* Rocha N, Neefjes J (2008) MHC class II molecules on the move for successful antigen presentation. EMBO J 27: $1-5$

Roy A, Kucukural A, Zhang Y (2010) I-TASSER: a unified platform for automated protein structure and function prediction. Nat Protoc 5:725-738

* Sangsuriya P, Huang JY, Chu YF, Phiwsaiya K and others (2014) Construction and application of a protein interaction map for white spot syndrome virus (WSSV). Mol Cell Proteomics 13:269-282

* Sangsuriya P, Charoensapsri W, Sutthangkul J, Senapin S, Hirono I, Tassanakajon A, Amparyup P (2018) A novel white spot syndrome virus protein WSSV164 controls prophenoloxidases, PmproPOs in shrimp melanization cascade. Dev Comp Immunol 86:109-117

* Schleicher TR, Yang J, Freudzon M, Rembisz A and others (2018) A mosquito salivary gland protein partially inhibits Plasmodium sporozoite cell traversal and transmission. Nat Commun 9:2908

* Sealy R, Chaka W, Surman S, Brown SA, Cresswell P, Hurwitz L (2008) Target peptide sequence within infectious human immunodeficiency virus type 1 does not ensure envelope-specific T-helper cell reactivation: influences of cysteine protease and gamma interferoninduced thiol reductase activities. Clin Vaccine Immunol 15:713-719

Singh R, Cresswell P (2010) Defective cross-presentation of viral antigens in GILT-free mice. Science 328: 1394-1398

Sinnathamby G, Maric M, Cresswell P, Eisenlohr LC (2004) Differential requirements for endosomal reduction in the presentation of two H2-E(d)-restricted epitopes from influenza hemagglutinin. J Immunol 172: 6607-6614

* Suraprasit S, Methatham T, Jaree P, Phiwsaiya K and others (2014) Anti-lipopolysaccharide factor isoform 3 from Penaeus monodon (ALFPm3) exhibits antiviral activity by interacting with WSSV structural proteins. Antiviral Res 110:142-150

*Tassanakajon A, Somboonwiwat K, Supungul P, Tang S (2013) Discovery of immune molecules and their crucial functions in shrimp immunity. Fish Shellfish Immunol 34: 954-967

*Teramoto T, Chiang $H$, Takhampunya R, Manzano M, Padmanabhan R, Maric M (2013) Gamma interferoninducible lysosomal thioreductase (GILT) ablation renders mouse fibroblasts sensitive to dengue virus replication. Virology 441:146-151

*Verbruggen B, Bickley LK, van Aerle R, Bateman KS, Stentiford GD, Santos EM, Tyler CR (2016) Molecular mechanisms of white spot syndrome virus infection and perspectives on treatments. Viruses 8:23

*Wunderlich M, Otto A, Maskos K, Mücke M, Seckler R, Glockshuber R (1995) Efficient catalysis of disulfide formation during protein folding with a single active-site cysteine. J Mol Biol 247:28-33

Yang J, Yan R, Roy A, Xu D, Poisson J, Zhang Y (2015) The I-TASSER Suite: protein structure and function prediction. Nat Methods 12:7-8 
Zhang Y (2008) I-TASSER server for protein 3D structure prediction. BMC Bioinformatics 9:40

Zhang D, Pan D, Cui S, Su T, Qiu L, Zhu C, Jiang S (2010) Molecular characterization and expression analysis of interferon- $\gamma$-inducible lysosomal thiol reductase (GILT) gene from pearl oyster Pinctada fucata. Dev Comp
Immunol 34:969-976

Khou Q, Xu L, Li H, Qi YP, Yang F (2009) Four major envelope proteins of white spot syndrome virus bind to form a complex. J Virol 83:4709-4712

Zhu YB, Li HY, Yang F (2006) Identification of an envelope protein (VP39) gene from shrimp white spot syndrome

Appendix.

Table A1. Locations of the domains/sites present in white spot syndrome virus (WSSV) proteins

\begin{tabular}{|c|c|c|}
\hline WSSV protein & Domains/sites & Amino acid residues \\
\hline \multirow[t]{4}{*}{ WSSV002 } & Low complexity & $24-35$ \\
\hline & Casein kinase II phosphorylation site & $2-5$ \\
\hline & Protein kinase $\mathrm{C}$ phosphorylation site & $32-34$ \\
\hline & $N$-myristoylation site & $12-17$ \\
\hline \multirow[t]{7}{*}{ WSSV164 } & Low complexity & $5-16,21-56,101-118,366-397$ \\
\hline & $N$-glycosylation site & $\begin{array}{c}5-8,50-53,102-105,120-123 \\
160-163,177-180,295-298\end{array}$ \\
\hline & $N$-myristoylation site & $\begin{array}{c}83-88,119-124,205-210,210-215 \\
386-391,388-393\end{array}$ \\
\hline & Casein kinase II phosphorylation site & $\begin{array}{c}77-80,122-125,123-126 \\
154-157,172-175,179-182 \\
217-220,222-225\end{array}$ \\
\hline & Protein kinase $\mathrm{C}$ phosphorylation site & $\begin{array}{c}106-108,111-113,136-138 \\
266-268\end{array}$ \\
\hline & Tyrosine kinase phosphorylation site & $324-332$ \\
\hline & $\begin{array}{l}\text { CAMP- and cGMP- dependent } \\
\text { protein kinase phosphorylation site }\end{array}$ & $108-111,143-146$ \\
\hline \multirow[t]{5}{*}{ WSSV189 } & Low complexity & $49-56$ \\
\hline & $N$-glycosylation site & $117-120,162-165,212-215$ \\
\hline & $N$-myristoylation site & $171-176,204-209$ \\
\hline & Casein kinase II phosphorylation site & $8-11,9-12,66-69$ \\
\hline & Protein kinase $\mathrm{C}$ phosphorylation site & $\begin{array}{c}8-10,119-121,145-147,163-165 \\
195-197,208-210\end{array}$ \\
\hline \multirow[t]{5}{*}{ WSSV471 } & Coiled-coil & $8-129$ \\
\hline & $N$-glycosylation site & $117-120$ \\
\hline & Casein kinase II phosphorylation site & $\begin{array}{c}6-9,25-28,34-37,38-41 \\
79-82,121-124\end{array}$ \\
\hline & Protein kinase $\mathrm{C}$ phosphorylation site & $79-81,94-96,118-120,121-123$ \\
\hline & Tyrosine kinase phosphorylation site & $5-12$ \\
\hline
\end{tabular}

Editorial responsibility: Stewart Johnson, Nanaimo, British Columbia, Canada
Submitted: October 16, 2018; Accepted: April 18, 2019 Proofs received from author(s): June 23, 2019 